\title{
Short-term outcomes following single-port laparoscopic surgery in elderly patients with colon cancer compared with younger patients
}

\author{
MITSUNOBU TAKEDA, MASAYOSHI TOKUOKA, HAJIME HIROSE, YOSHIHITO IDE, \\ YASUJI HASHIMOTO, JIN MATSUYAMA, SHIGEKAZU YOKOYAMA, TAKASHI MORIMOTO, \\ YUKIO FUKISHIMA, TAKASHI NOMURA, KEN KODAMA and YO SASAKI \\ Department of Surgery, Yao Municipal Hospital, Yao, Osaka 581-0069, Japan
}

Received July 27, 2017 ; Accepted February 3, 2017

DOI: $10.3892 / \mathrm{ol} .2017 .6344$

\begin{abstract}
Surgeons are increasingly being faced with the challenge of treating elderly patients with colon cancer. The present study therefore aimed to compare the short-term outcomes of single-port laparoscopic surgery (SILS) for elderly patients with colon cancer ( $\geq 70$ years) with those in younger patients (41-69 years; control group). Among 100 patients with colorectal cancer who had been treated with single-port laparoscopic surgery between January 2011 and December 2014, $56(56.0 \%)$ were $\geq 70$ years of age. The results of treatment and short-term outcomes in the elderly group $(n=56)$ were retrospectively compared with the younger patients in the control group $(n=44)$. The sex distribution, body mass index, history of prior surgery and the American Society of Anesthesiologists physical status classification were similar between the groups. Onodera's prognostic nutritional index demonstrated significant differences between the elderly and control groups (38.3 vs. 49.8; $\mathrm{P}<0.05)$. No significant differences were observed in the mean length of surgery $(219.5 \pm 73.5 \mathrm{vs} .201 .4 \pm 76.5 \mathrm{~min}$; $\mathrm{P}=0.43)$, estimated blood loss $(32.2 \pm 74.5$ vs. $36.1 \pm 90.2 \mathrm{ml}$; $\mathrm{P}=0.10)$, postoperative complications (10.9 vs. $7.1 \% ; \mathrm{P}=0.78)$, length of postoperative hospital stay $(9.6 \pm 12.5$ vs. $7.3 \pm 3.0$ days; $\mathrm{P}=0.23)$ or number of harvested lymph nodes $(21.8 \pm 24.3$ vs. $22.5 \pm 11.3 ; \mathrm{P}=0.87$ ) between the elderly and control groups. In conclusion, the results of the present study demonstrate that SILS may be carried out feasibly in elderly patients with colon cancer.
\end{abstract}

\section{Introduction}

Colorectal cancer is the fourth most common type of cancer in males and the third most common type of cancer in females

Correspondence to: Dr Masayoshi Tokuoka, Department of Surgery, Yao Municipal Hospital, 1-3-1 Ryuge, Yao, Osaka 581-0069, Japan

E-mail: mtokuoka1017@yahoo.co.jp

Key words: single-port laparoscopic surgery, elderly patients, single-incision laparoscopic colectomy worldwide, and in numerous developed countries it is the second leading cause of cancer-associated mortality (1). In addition, colon cancer is a major cause of mortality in Japan (2). An aging population and an increased life expectancy worldwide have led to an increased incidence rate of colorectal cancer in elderly patients, for whom treatment is often complicated (3).

The predicted mortality and type of radical surgery should be taken into consideration when considering surgical options for elderly patients, since elderly patients have an increased number of preoperative risk factors compared with younger patients. Therefore, minimally invasive surgery with a limited number of risk factors is important for elderly patients. Single-incision laparoscopic surgery (SILS) is a more recent innovation proposed to further the outcomes of multi-port laparoscopy (4). SILS was first reported in 1999 for cholecystectomy and then applied to colorectal resection in previous studies (4-6). SILS has been successfully used as a method for colectomy (7). However, the feasibility of SILS in elderly patients with colon cancer has not been previously demonstrated, to the best of our knowledge. The purpose of the present retrospective study was to elucidate the feasibility of SILS for elderly patients.

\section{Materials and methods}

Patient information. Between January 2011 and December 2014, 100 patients underwent SILS for the treatment of colon cancer at Yao Municipal Hospital (Yao, Osaka, Japan). The present study reports the results of a retrospective case controlled study of these 100 patients who underwent elective surgical intervention for the treatment of colon cancer. The elderly group consisted of 56 patients who were $\geq 70$ years (elderly group; mean age, 76.6 years). The control group consisted of 44 patients, all $<70$ years (mean age, 61.4 years).

All procedures were performed by colorectal surgeons who were experienced in multi-port laparoscopic surgery or standard laparoscopic surgery (8). Data on patient demographics, body mass index (BMI), American Society of Anesthesiologists physical status (ASA-PS) classification (9), Onodera's prognostic nutritional index (PNI) (10), tumor location, tumor size, personal history of surgery, surgery duration, estimated blood loss, number of lymph nodes harvested, length of the resected margin, conversion into open surgery, insertion of an 
additional port, perioperative complications, morbidity and the length of hospital stay were compared between the elderly and control groups. Onodera et al (10) first reported the validity of Onodera's PNI to predict the prognosis of 189 gastrointestinal surgical patients. Onodera's PNI is an equation which takes into account the levels of serum albumin and total lymphocyte count. Onodera's PNI can be applied rapidly in a large number of patients. Validation of Onodera's PNI has been applied for patients with colon cancer (11).

Surgical technique. The surgery was performed as described in a previous study (12). Under general anesthesia, the patient was placed in the modified lithotomy position. A wound protector (Lap protector LP ${ }^{\mathrm{TM}}$; Hakkou Shoji, Chikuma, Nagano, Japan) was inserted through a 30-mm transumbilical incision. A single port access device (EZ-access ${ }^{\mathrm{TM}}$, Hakkou Shoji) was then mounted to the wound protector and three 5 -mm ports were placed (Fig. 1). The surgical procedures and instruments used were the same as those used for standard laparoscopic surgery with a flexible 5-mm scope (Olympus Medical Systems Corp., Tokyo, Japan).

An additional incision or trocar port was placed if necessary, in order to complete the procedure, and conversion into open laparotomy was maintained as an option. The decision to use an additional trocar or to convert into open laparotomy was subject to the opinion of the surgeon performing the procedure. For lymphadenectomy, a complete mesocolic excision (CME) with central vascular ligation (CVL) was performed. The final incision was extended to a length comparable with the size of the specimen.

Right hemicolectomy. For the right hemicolectomy, the patient was placed in the Trendelenburg semi-left lateral position. The surgeon and camera operator stood on the left side of the patient. The procedure was performed via an inferior approach, with the initial peritoneal dissection occurring between the mesoileum and the retroperitoneum. Following mesocolic plane resection, the duodenum and pancreas were sufficiently exposed, and the ileocolic, right colic (if necessary) and middle colic vessels were ligated and dissected between clips at their origin to allow CME and CVL (Fig. 2). Subsequent to the dissection of the greater omentum, the hepatic flexure was mobilized. The specimen was retrieved via the transumbilical incision, and an extracorporeal functional end-to-end anastomosis was subsequently performed.

Sigmoidectomy and anterior resection. The patient was placed in the Trendelenburg semi-right lateral position. The surgeon and camera operator stood on the right side of the patient. The procedure was performed via a standard medial-to-lateral approach. The inferior mesenteric artery and the inferior mesenteric vein were skeletonized, clipped, and divided for CME and CVL (Fig. 3). Surgeons then dissected downwards from the mesenteric window to the pelvis. The sigmoid colon up to the splenic flexure was then mobilized. The descending colon and sigmoid colon was pulled anteromedially to ensure preservation of the left ureter and gonadal vessels. The fat surrounding the rectum for $>50 \mathrm{~mm}$ distal to the lesion was dissected and subsequently transected normally using an endoscopic linear stapler (Endo GIA ${ }^{\mathrm{TM}}$; Covidien LLC, Mansfield,

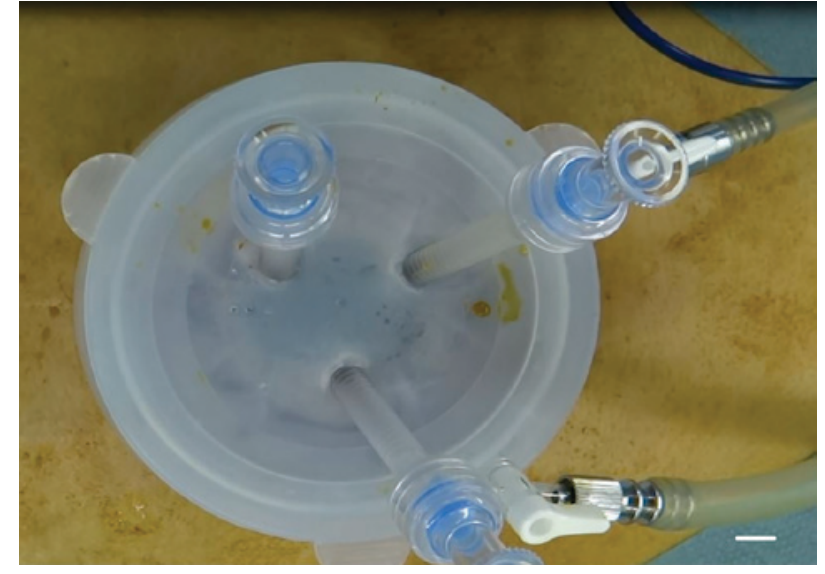

Figure 1. EZ-access was mounted onto the Lap protector mini and three 5 -mm ports were made in the EZ-access. Scale bar: $5 \mathrm{~mm}$.

MA, USA) with a purple cartridge inserted from the umbilical port with a 12-mm EZ Link (Hakko Co. Ltd., Nagano, Japan). The specimen was retrieved through the umbilical incision, and the double stapling technique was applied for anastomosis.

Statistical analysis. Statistical analysis was performed using SPSS software (version 14.0; SPSS, Inc., Chicago, IL, USA). All variables were evaluated using a $\chi^{2}$ test or Student's t-test, as appropriate. $\mathrm{P}<0.05$ was considered to indicate a statistically significant difference.

\section{Results}

In the elderly patient group and the control group, the primary tumor sites included the cecum $(n=7 ; n=4)$, ascending colon $(n=24 ; n=6)$, descending colon $(n=1 ; n=2)$, sigmoid colon $(n=14$; $n=27)$ and the rectosigmoid colon $(n=10 ; n=5)$, respectively (Table I). The primary tumor location in the elderly group was more frequently in the right colon. In the control group, the primary tumor location was more frequently in the left colon.

The median BMI of the elderly and control groups were $22.8 \pm 3.7$ and $23.2 \pm 4.0 \mathrm{~kg} / \mathrm{m}^{2}$, respectively. The sex distribution $(\mathrm{P}=0.86)$, history of prior surgery ( 20 vs. $13 ; \mathrm{P}=0.73)$, and ASA-PS $(\mathrm{P}=0.42)$ were not significantly different between the groups. Renal function, respiratory function and cardiac function were poorer in the elderly group compared with the control group; however, no significant differences were identified.

Onodera's PNI was significantly decreased in the elderly compared with the control group ( 38.3 vs. $49.8 ; \mathrm{P}<0.05)$ ). For 1 patient $(1.8 \%)$ in the elderly group, surgery was converted into laparotomy, since it was not possible to make free working space in the abdominal cavity due to bone deformities caused by spondylocase. The remaining 99 patients underwent curative colectomy without conversion into multi-port laparoscopic surgery or open surgery. In the elderly group, 31 patients underwent right hemicolectomy and 25 underwent sigmoid colectomy and anterior resection. In the control group, 10 patients underwent right hemicolectomy and 34 underwent sigmoid colectomy and anterior resection. The mean skin incision length in the elderly and control group was $34.0 \pm 5.9$ and $35.5 \pm 6.8 \mathrm{~mm}$, respectively. The mean surgical duration in the elderly and control group was $219.5 \pm 73.5$ and $201.4 \pm 76.5 \mathrm{~min}$, 

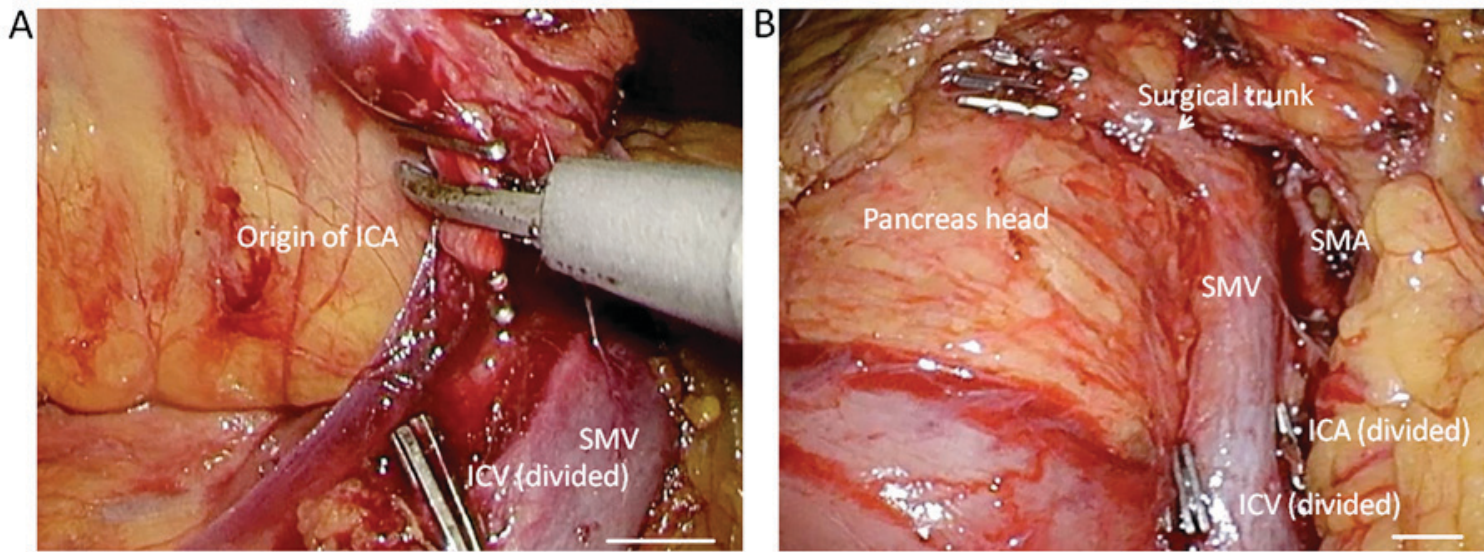

Figure 2. Surgical procedure for right hemicolectomy with complete mesocolic excision for right-sided colon cancer at Yao Municipal Hospital (Osaka, Japan). (A) Exposure and ligation of the origin of the ICA and ICV by complete mesocolic excision with dissection of the entire right-side mesocolon. (B) Completion of the lymphadenectomy with complete mesocolic excision with central vascular ligation for right-sided colon cancer. ICA, ileocolic artery; ICV, ileocolic vein; SMA, superior mesenteric artery; SMV, superior mesenteric vein. Scale bars: $5 \mathrm{~mm}$.
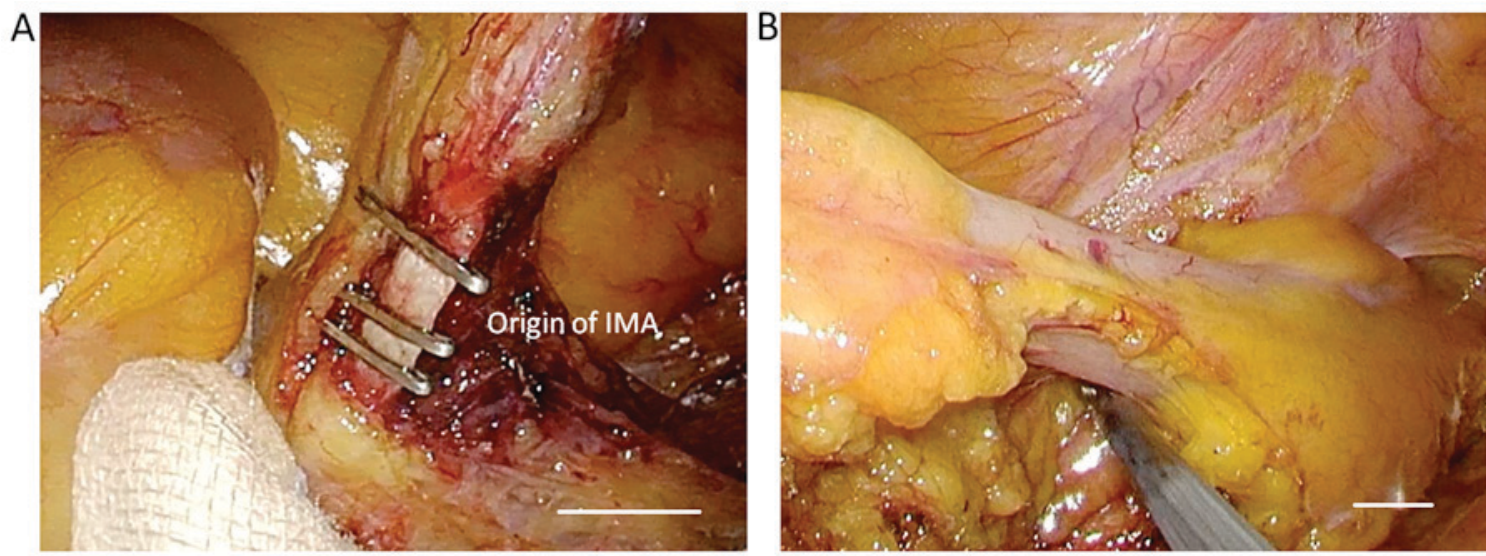

Figure 3. Surgical procedure for sigmoidectomy with complete mesocolic excision for left-sided colon cancer at Yao Municipal Hospital (Osaka, Japan), (A) Exposure and ligation of the origin of the IMA in complete mesocolic excision with central vascular ligation for left-sided colon cancer. (B) The fat surrounding the colon $>50 \mathrm{~mm}$ distal to the lesion was removed and transected intracorporeally. IMA, inferior mesenteric artery. Scale bars: $5 \mathrm{~mm}$.

respectively, and blood loss was $32.2 \pm 74.5$ and $36.1 \pm 90.2 \mathrm{ml}$, respectively. The mean number of harvested lymph nodes in the elderly and control group was $21.8 \pm 24.3$ and $22.5 \pm 11.3$, respectively. No intraoperative complications occurred in either group.

Postoperative complications included an anastomotic leak, wound infection, pneumonia, deep vein thrombosis, dysuria, ileus and urinary tract infections. Postoperative complications occurred in 6 cases (10.7\%) in the elderly group and 3 cases (6.81\%) in the control group. In the elderly group, 14 patients (25.0\%) were $>80$ years old; however, serious complications were not observed in these patients, and delirium was not observed in any patient from either group. The time until the first production of feces following surgery in the elderly and control group was $4.11 \pm 4.60$ and $3.80 \pm 1.40$ days, respectively. The patients were discharged following a mean period of $9.60 \pm 12.5$ and $7.30 \pm 3.0$ days postoperatively in the elderly and control groups, respectively (Table II). No mortality occurred in any patient from either group, and there was no readmission within 30 days postoperatively. Regarding oncological features, the number of resected lymph nodes and resection margins and tumor size did not differ significantly between the elderly and control groups. There were also no recurrences in either group $<1$ year following surgery, according to follow-up physical examinations every 3 months and computed tomography scans performed every 6 months.

\section{Discussion}

Currently, numerous surgeons have adopted laparoscopic techniques. The oncological safety of laparoscopic colectomy in patients with cancer has been demonstrated in randomized trials (13). As a result, laparoscopic surgery has steadily become a safe and practical treatment option even for malignant diseases of the colon and rectum.

The number of elderly patients with colorectal cancer requiring surgery is increasing annually, due to an increase in life expectancy and therefore an increasingly elderly population. According to the census figures in 2010, the average lifespan is 79.64 years for males and 86.39 years for females in Japan (14). The proportion of elderly individuals who are $\geq 70$ years in Japan is $16.4 \%$, and this number is increasing (15). 


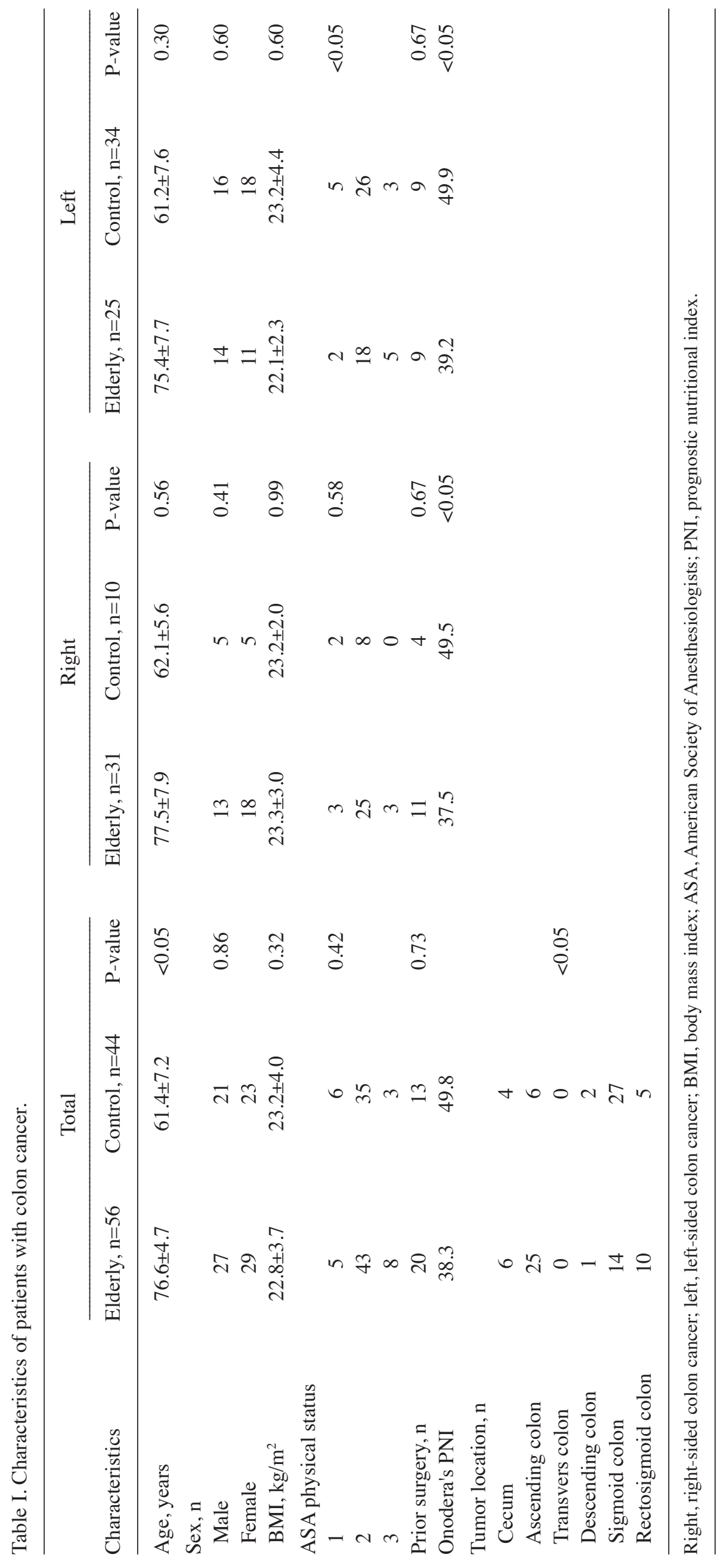




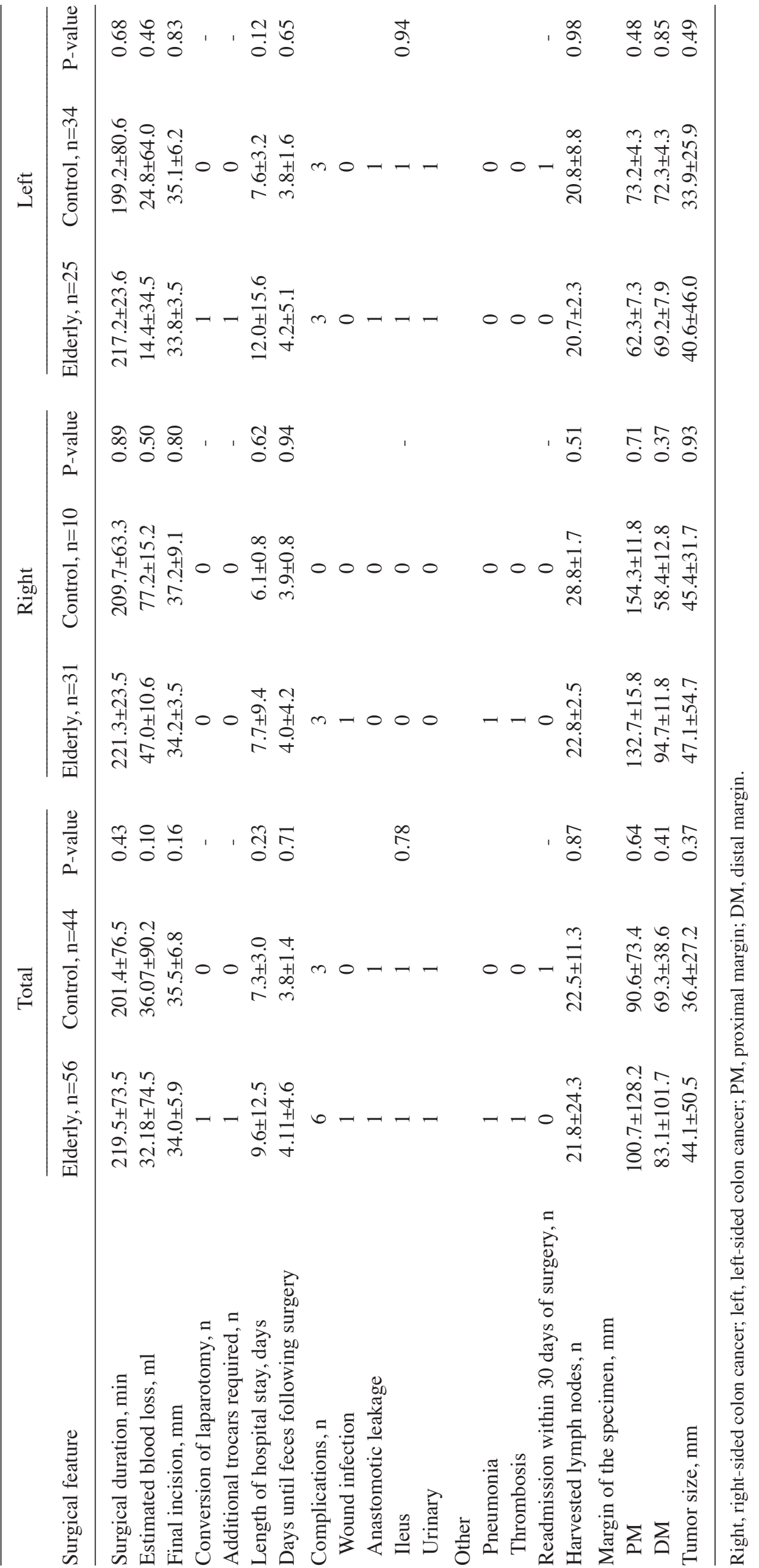


The number of cases of elderly patients requiring surgery is expected to increase. It is clear that, as patients age, there is an associated increase in comorbid conditions that often complicate the perioperative management of elderly patients who undergo major surgery.

The most important part of surgical practices for the treatment of elderly patients with a malignant disease is to balance the invasiveness of treatment and radical cure, which are often conflicting. Highly invasive surgery can only be performed in patients with a high tolerability. Previous studies indicate that lymph node dissection is less common in elderly patients and that there is a tendency to avoid invasive surgery in elderly patients $(16,17)$. However, to the best of our knowledge, there is no reported difference in cancer aggressiveness between younger patients and elderly patients. Previous reports indicate that radical surgery for elderly patients with colorectal cancer can improve the prognosis (18) and that radical surgery should be performed for elderly patients as it is for younger patients, provided that the main organs are functional (19).

A previous study demonstrated that multi-port laparoscopic-assisted colectomy in elderly patients is safe and feasible (6), and has numerous benefits over open colectomy since it appears to be less physiologically stressful compared with conventional open laparotomy. Multi-port laparoscopic assisted colectomy should be considered as the preferred surgical approach in the management of numerous colorectal diseases for appropriately selected elderly patients (6).

At Yao Municipal Hospital, it is considered that less-invasive surgery should be performed in elderly patients as often as possible while aiming for a radical cure and considering the functional decline of the main organs. As a result, the authors introduced a multi-port laparoscope-assisted surgery in 2009 in the Department of Surgery. Furthermore, according to the theory of reduced-port surgery (20-23), single-incision laparoscopic colectomy (SILC) was introduced to Yao Municipal Hospital in July 2013 with the aim of providing a less-invasive surgical technique.

Reduced port surgery aims to decrease the size and number of ports to preserve the view afforded by the laparoscope while making the surgery less invasive. Previous studies have successfully discussed the advantages of decreasing the number of laparoscopic ports, including improved cosmetic results, decreased postoperative pain and shorter recovery period; however, there is a technical complexity to decreased number of ports, including instrument crowding and insufficient counter traction (20-23).

SILS as a treatment for colon cancer has been preliminary described by Bucher et al (6), and through additional case reports and small case series (24-27). Previously, Papaconstantinou et al (28) described that, when compared with established laparoscopic techniques, SILC can improve patient recovery through a decrease in early postoperative pain and shorter length of hospital stay. However, other than the cosmetic advantage, the benefits of SILS are controversial.

In the present study, no statistically significant differences were identified between the short-term outcomes for the elderly and control groups of patients with colon cancer who underwent SILS. To the best of our knowledge, the present study is the first reported case-controlled study investigating colon cancer and it involved elderly patients (mean age, 76.6 years).
The present study demonstrated that the short-term outcomes of SILS in elderly ( $\geq 70$ years) and younger ( $<70$ years) patients are equivalent (Table II). The overall rate of morbidity and the number of cases with postoperative complications were almost equivalent in the two groups, and the length of hospital stay and the time until first passing of feces subsequent to surgery did not differ significantly between the two groups.

It has been reported that Onodera's PNI may be used as a prognostic factor of colorectal cancer (11). In the present study, Onodera's PNI score in the elderly patient group was significant lower compared with the control group (38.3 vs. 49.8; $\mathrm{P}<0.05$ ), whereas the frequency of postoperative complications was similar between the two groups. The results of the present study suggested that SILS in elderly patients is equivalent to that for younger patients.

With regard to the oncological quality of the surgery, the number of resected lymph nodes and resection margins did not differ significantly between the elderly and control groups. The mean number of removed lymph nodes was between 20.7 (elderly group) and 28.8 (control group), which is consistent with what has been revealed in other studies $(28,29)$. The total surgical conversion rate for the 100 patients with colon cancer was $1.0 \%$, which is consistent with previous studies $(29,30)$. Therefore, radical surgery may be performed for elderly patients as it is for younger patients, which may improve prognosis (17).

The single-incision laparoscopic approach used in the present study may have contributed to these low complication rates, and the results of the present study demonstrate that age alone should not be considered a reason to withhold surgical treatment from elderly patients regarding morbidity and mortality.

The results of the present study indicate that SILS is oncologically feasible, and that a curative procedure for elderly patients with colon cancer is equivalent to that for younger patients. These results are consistent with those of previous studies in the general population. Therefore, it is considered that these results justify the use of SILS in the routine treatment of elderly patients with colon cancer.

\section{References}

1. Center MM, Jemal A and Ward E: International trends in colorectal cancer incidence rates. Cancer Epidemiol Biomarkers Prev 18: 1688-1694, 2009.

2. World Cancer Research Fund and American Institute for Cancer Research Food, Nutrition, Physical Activity and the Prevention of Cancer: A Global Perspective. Washington, DC: American Institute for Cancer Research; 2007.

3. Fiscon V, Portale G, Migliorini G and Frigo F: Laparoscopic resection of colorectal cancer in elderly patients. Tumori 96: 704-708, 2010.

4. Piskun G and Rajpal S: Transumbilical laparoscopic cholecystectomy utilizes no incisions outside the umbilicus. J Laparoendosc Adv Surg Tech A 9: 361-364, 1999.

5. Remzi FH, Kirat HT, Kaouk JH and Geisler DP: Single-port laparoscopy in colorectal surgery. Colorectal Dis 10: 823-826, 2008.

6. Bucher P, Pugin F and Morel P: Single port access laparoscopic right hemicolectomy. Int J Colorectal Dis 23: 1013-1016, 2008.

7. Jacob BP and Salky B. Laparoscopic colectomy for colon adenocarcinoma: An 11-year retrospective review with 5-year survival rates. Surg Endosc 19: 643-649, 2005.

8. Hinoi T, Kawaguchi Y, Hattori M, Okajima M, Ohdan H, Yamamoto S, Hasegawa H, Horie H, Murata K, Yamaguchi S, et al: Laparoscopic versus open surgery for colorectal cancer in elderly patients: A multicenter matched case-control study. Ann Surg Oncol 22: 2040-2250, 2015. 
9. Saklad M: Grading of patients for surgical procedures. Anesthesiol 2: 281-284, 1941.

10. Onodera T, Goseki N and Kosaki G: Prognostic nutritional index in gastrointestinal surgery of malnourished cancer patients. Nippon Geka Gakkai Zasshi 85: 1001-1005, 1984 (In Japanese).

11. Nozoe T, Kohno M, Iguchi T, Mori E, Maeda T, Matsukuma A and Ezaki T: The prognostic nutritional index can be a prognostic indicator in colorectal carcinoma. Surg Today 42: 532-535, 2012.

12. Tokuoka M, Ide Y, Takeda M, Hashimoto Y, Matsuyama J, Yokoyama S, Morimoto T, Fukushima Y, Nomura T, Kodama K and Sasaki Y: Single-incision Plus one port laparoscopic total mesorectal excision and bilateral pelvic node dissection for advanced rectal cancer-a medial umbilical ligament approach. Int Surg 100: 417-422, 2015.

13. Lacy AM, García-Valdecasas JC, Delgado S, Castells A, Taurá P, Piqué JM and Visa J: Laparoscopy-assisted colectomy versus open colectomy for treatment of non-metastatic colon cancer: A randomaised trial. Lancet 359: 2224-2229, 2002

14. Index mundi: http://www.indexmundi.com/facts/japan/ life-expectancy-at-birth. Accessed August 17, 2016.

15. POPULATION AND HOUSEHOLDS OF JAPAN 2010: http:// www.stat.go.jp/english/data/kokusei/2010/poj/mokuji.htm. Accessed August 17, 2016.

16. Satou S, Yoneda K, Ogata J, Mori M, Wada T, Sakamoto N Majima A, Katou K, Aoki T and Koyanagi Y: Clinicopathological feature and prognosis of colorectal cancer in elderly-people ( 75 or more years old). Journal of Japan Society of Coloproctology 56 41-47, 2003.

17. Fujii S, Ishibe A, Ota M, Watanabe K, Watanabe J, Tatsumi K, Yamagishi S, Ichikawa Y, Ohki S, et al: Short-term and long-term outcomes of colorectal cancer surgery in elderly patients (80 years or more) and measures for improving treatment results Nippon Daicho Komonbyo Gakkai Zasshi 66: 221-228, 2013 (In Japanese).

18. Man-I M, Nishisho I, Mishima H, Yagyu T, Kikkawa N and Tsujinaka T: Risk factors of postoperative complication and prognostic factors in aged patients with resectable colorectal carcinomas by using multivariate analysis. Jpn J Gastroenterol Surg 35: 590-597, 2002

19. Nakamura T, Mitomi H, Onozato W, Sato T, Ikeda A, Naito M, Ogura N, Kamata H, Ooki A and Watanabe M: Oncological outcomes of laparoscopic surgery in elderly patients with colon cancer: A comparison of patients 64 years or with those 75 years or older. Hepatogastroenterology 58: 1200-1204, 2011.
20. Chen WT, Chang SC, Chiang HC, Lo WY, Jeng LB, Wu C and Ke TW: Single-incision laparoscopic versus conventional laparoscopic right hemicolectomy: A comparison of short-term surgical results. Surg Endosc 25: 1887-1892, 2011.

21. Champagne BJ, Lee EC, Leblanc F, Stein SL and Delaney CP: Single-incision vs straight laparoscopic segmental colectomy: A case-controlled study. Dis Colon Rectum 54: 183-186, 2011.

22. Champagne BJ, Papaconstantinou HT, Parmar SS, Nagle DA, Young-Fadok TM, Lee EC and Delaney CP: Single-incision versus standard multiport laparoscopic colectomy: A multicenter, case-controlled comparison. Ann Surg 255: 66-69, 2012.

23. Poon JT, Cheung CW, Fan JK, Lo OS and Law WL: Single-incision versus conventional laparoscopic colectomy for colonic neoplasm: A randomized, controlled trial. Surg Endosc 26: 2729-2734, 2012.

24. Takemasa I, Sekimoto M, Ikeda M, Mizushima T, Yamamoto H, Doki Y and Mori M: Transumbilical single-incision laparoscopic surgery for sigmoid colon cancer. Surg Endosc 24: 2321, 2010.

25. Ramos-Valadez DI, Patel CB, Ragupathi M, Bartley Pickron T and Haas EM: Single incision laparoscopic right hemicolectomy: Safety and feasibility in a series of consecutive cases. Surg Endosc 24: 2613-2616, 2010.

26. Hirano Y, Hattori M, Kitamura H, Maeda K, Douden K and Hashizume Y: Hybrid single-incision laparoscopic sigmoidectomy: The effective use of small incision. J Laparoendosc Adv Surg Tech A 21: 625-627, 2011.

27. Katsuno G, Fukunaga M, Nagakari K, Yoshikawa S, Ouchi M and Hirasaki Y: Single-incision laparoscopic colectomy for colon cancer: Early experience with 31 cases. Dis Colon Rectum 54: 705-710, 2011.

28. Papaconstantinou HT, Sharp N and Thomas JS: Single-incision laparoscopic right colectomy: A case-matched comparison with standard laparoscopic and hand-assisted laparoscopic techniques. J Am Coll Surg 213: 72-82, 2011.

29. Keller DS, Flores-Gonzalez JR, Ibarra S and Haas EM: Review of 500 single incision laparoscopic colorectal surgery cases - Lessons learned. World J Gastroenterol 22: 659-667, 2016.

30. Veldkamp R, Gholghesaei M, Bonjer HJ, Meijer DW, Buunen M, Jeekel J, Anderberg B, Cuesta MA, Cuschierl A, Fingerhut A, et al: Laparoscopic resection of colon Cancer: consensus of the European Association of Endoscopic Surgery (EAES). Surg Endosc 18: 1163-1185, 2004. 